\title{
Detecting Shrew HTTP Flood Attacks for Flash Crowds
}

\author{
Yi Xie and Shun-Zheng Yu \\ Department of Electrical and Communication Engineering Sun Yat-Sen University, \\ Guangzhou 510275, P.R. China \\ xieyicn@163.com
}

\begin{abstract}
Countering network attacks is becoming ever more challenging. Web-based vulnerabilities represent a substantial portion of the security exposures of computer networks. In order to detect a new Web-based assault named shrew Distributed Denial of Service attacks based on HTTP flood, Principle Component Analysis and Independent Component Analysis are applied to abstract the multivariate observation vector. A novel anomaly detector based on hidden semi-Markov model is proposed. Experiment results based on real traffic trace and emulated attacks show, the scheme can be used effectively to implement the detection of the shrew HTTP flood attacks embedded in the normal flash crowd of large-scale Website; and the detection is not dependent on the intensity of attack traffic.
\end{abstract}

\section{Introduction}

In the past ten years, Web-based applications have become a popular way to provide information and services dynamically. Electronic commerce, ranging from internet shopping malls to Web-based banking and trading, has become an integral component of modern society. At the same time, Web servers and Web-based applications are popular attack targets. CVE [1] reported Web-based vulnerabilities accounted for more than $25 \%$ of the total number of security flaws during 1999 to 2005 .

For these reasons, studies have been done to detect Web-based attacks. However, it isn't enough for most current works to detect the special new Web-based attacks known as "Application layer Distribute Denial of Service" (App-DDoS) attacks. The main difference between the App-DDoS attacks and the traditional DDoS attacks is that they are protocol-compliant, non-intrusive, and utilize legitimate Web requests to overwhelm system resources. App-DDoS attacks utilize the weakness enabled by the standard practice of opening services such as HTTP (TCP port 80) and HTTPS (TCP port 443) through most firewalls to launch the flood. Many applications and protocols, both legitimate and illegitimate, can use these openings to tunnel through firewalls by connecting over standard TCP port 80 (e.g., Code Red virus) or encapsulating in SSL tunnels (HTTPS). Attack packets aiming at these services pass through the firewall without being identified. Thus, most current intrusion detections designed for Web-based attacks and DDoS attacks become invalid when they are used to block this type of sophisticated attacks. This is especially the case when the attackers secrete the offensive traffic in the flash crowds of special heavily-accessed Web server environments (e.g., Olympic Games Website, e-commerce Website). 
This paper focuses on the detection of shrew HTTP flood attacks under unstable and bursty background traffic (e.g., flash crowd). Shrew HTTP flood is a special type of App-DDoS attacks which is a compound of shrew attack and HTTP flood. The difficulties of its detection include: (i) in stead of constantly injecting traffic flows with huge rates into the network, shrew attackers launch assaults by sending burst pulses periodically, which has high peak rate while maintaining a low average rate to exhibit "stealthy" behavior; and (ii) burst traffic and high volume are the common characteristics of HTTP flood and flash crowd, which make the detection much more difficult. This paper meets this challenge by a novel detection scheme.

We carry out the anomaly detection by file popularity which has been proved to have significant temporal stability in [7]. The main contributions of this paper are (i) development of a new efficient mechanism to detect the shrew HTTP flood attacks which mimic (or mix with) the normal flash crowds and utilizes legitimate requests to overwhelm system resources, (ii) applying principal component analysis (PCA) [8] and independent component analysis (ICA) [9] to abstract multivariate observation vector, (iii) using hidden semi-Markov model (HsMM) [10] to construct the anomaly recognizer, and (iv) experimentation of a real traffic of heavily-accessed Web server and an emulated shrew HTTP flood attack to evaluate the model.

The rest of the paper is organized as follows. In section 2, we describe related work in Web-based attacks detection. In section 3, we present the rationale of this work. In section 4, we conduct experiments to validate our detection algorithms. Finally, in section 5, we conclude our work.

\section{Related Work}

The detection of Web-based attacks has recently received considerable attention because of the increasingly critical role that Web-based services are playing, e.g., [3][4]. However, current detection systems are mostly misuse-based and therefore suffer from the problem of not being able to detect new attacks. Recently, Juan [5] accomplished the detection by analyzing each incoming HTTP payloads, which may lead to high computational complexity. In [6], Tombini proposed a serial architecture where Web-related events are first passed through an anomaly detection component. Then, the events that are identified as neither normal nor intrusive are passed on to a misuse detection component, but it requires extensive manual analysis to evaluate the characteristics of the events being analyzed.

A valuable recent work on App-DDoS can be found in [2][11]. In [2], the authors developed a counter-mechanism which assigns a suspicion measure to a session in proportion to its deviation from legitimate behavior. However, this work impliedly assumes the incoming traffic is stable, which is not always true in large-scale heavily-accessed Websites. In [11], Kandula et al. designed a system to protect a Web cluster from DDoS attacks by designing a probabilistic authentication mechanism using "puzzles". Unfortunately, requiring all users to solve graph puzzles has the possibility of annoying users and introducing additional service delays for legitimate users. This also has the effect of denying Web crawlers access to the site and as a result search engines may not be able to index the content. Finally, new techniques may render the graphical puzzles solvable using automated methods [12]. Other researchers have explored the defense of shrew attacks, e.g., [13]. However, authors of these 
literatures merely aimed at the TCP attacks in stead of HTTP flooding attacks. Furthermore, in contrast to our burst and unstable background traffic of detection scenario, their common implied hypothesis is that the background traffic is stable, which may not help in this paper.

\section{Detection Algorithms}

In contrast to the prior work, the detection scheme proposed in this paper uses passive detection which applies statistical methods to detect shrew HTTP flood attacks. Our observation data is file popularity which can be derived from the Web server log easily. In order to perform efficient, unsupervised, learning-based anomaly detection, this paper proposes a novel detection scheme based on multivariate statistical analysis and outlier detection. PCA and ICA are used to abstract the observed data, and then the HsMM is applied to construct the anomaly detector.

\subsection{File Popularity Matrix}

We define the $N \times T$ dimensional file popularity matrix as:

$$
A_{N \times T}=\left[\begin{array}{cccc}
a_{11} & a_{12} & \cdots & a_{1 T} \\
a_{21} & a_{22} & \cdots & a_{2 T} \\
\cdots & \cdots & \cdots & \cdots \\
a_{N 1} & a_{N 2} & \cdots & a_{N T}
\end{array}\right]=\left[\begin{array}{c}
\mathbf{a}_{1} \\
\mathbf{a}_{2} \\
\cdots \\
\mathbf{a}_{N}
\end{array}\right]=\left[\begin{array}{c}
\vec{a}_{1} \\
\vec{a}_{2} \\
\cdots \\
\vec{a}_{T}
\end{array}\right]^{\mathrm{T}}
$$

where $a_{i t}$ denotes the popularity of the $i^{\text {th }}$ document at $t^{\text {th }}$ time unit, $\mathbf{a}_{i}=\left[\begin{array}{lll}a_{i 1} & \cdots & a_{i T}\end{array}\right]$ is the time series of $i^{\text {th }}$ document's popularity, $\vec{a}_{t}=\left[\begin{array}{lll}a_{1 t} & \cdots & a_{N t}\end{array}\right]^{\mathrm{T}}$ denotes the popularities of $N$ documents at $t^{\text {th }}$ time unit, which forms the $N$-dimensional observation vector. Especially, if we consider the documents as a subspace and time as another subspace, the file popularity matrix reflects the spatial-temporal patterns of all users' access behaviors. Distribution of $\vec{a}_{t}$ presents the spatial distribution of popularity at $t^{\text {th }}$ time unit while the distribution of $a_{i}$ presents the temporal distribution of the $i^{\text {th }}$ document's popularity. For brevity of notation, we call them spatial distribution and temporal distribution, respectively.

In [7], the authors have found, although the domains from which clients access the popular content are unstable, there is significant temporal stability in file popularity of busy Website. Thus, file popularity is used as detection signal in this paper.

\subsection{HsMM}

Because of the high dimensional document space $(N)$, we can not monitor the shrew HTTP flood attacks merely by checking every document's popularity distribution. Furthermore, detection through checking popularity distribution of each document solely may give wrong warning signals because changes of individual document's popularity distribution don't imply attacks. Hence, we consider the joint distribution of $\mathrm{N}$-dimensional document space to implement numerical and effective detection in this paper. We extend the HsMM [10] to capture the dynamics of file popularity matrix and monitor the shrew HTTP flood attacks during flash crowd event. 
HsMM is a Hidden Markov Model (HMM) with variable state duration. It has been widely used in recognition and detection because it is very suitable to describe most practical physical signals (e.g., speech recognition, character recognition and DNA sequences clustering). The HsMM is a stochastic finite state machine, specified by $(S, \pi, A, P)$ where $S$ is a discrete set of hidden states with cardinality $M ; \pi$ is the probability distribution for the initial state $\pi_{i}=\operatorname{Pr}\left[s_{1}=i\right]$ and satisfy $\sum_{i} \pi_{i}=1, s_{t}$ denotes the state that the system takes at time $t$ and $i \in S ; A$ is the state transition matrix with probabilities $a_{i j}=\operatorname{Pr}\left[s_{t}=j \mid s_{t-1}=i\right]$ and satisfy $\sum_{j} a_{i j}=1, i, j \in S ; P$ is the state duration matrix with probabilities $p_{i}(d) \equiv \operatorname{Pr}\left[\tau_{t}=d \mid s_{t}=i\right]$ and satisfy $\sum_{d} p_{i}(d)=1, \tau_{t}$ denotes the remaining (or residual) time of the current state $s_{t}, i \in S, d \in\{1, \ldots, D\}, D$ is the maximum interval between any two consecutive state transitions. For brevity of notation, we denote the complete set of model parameters: $\lambda=\left(\left\{\pi_{i}\right\},\left\{a_{i j}\right\},\left\{b_{i}(k)\right\},\left\{p_{i}(d)\right\}\right)$. The entropy $(E n)$ of observation fitting to the HsMM and the average logarithmic entropy $(A L E)$ per observation is defined as the measure criteria as following, respectively:

$$
\begin{gathered}
E n=\operatorname{Pr}\left[\vec{o}_{1}^{T} \mid \lambda\right]=\sum_{i, d} \operatorname{Pr}\left[\vec{o}_{1}^{T},\left(s_{T}, \tau_{T}\right)=(i, d) \mid \lambda\right], \\
A L E=\ln \left(\operatorname{Pr}\left[\vec{o}_{1}^{T} \mid \lambda\right]\right) / T,
\end{gathered}
$$

In our previous work [10], we have developed efficient parameter reestimate algorithm and dynamic update algorithm for HsMM. Based on the work, HsMM is applied to construct the anomaly detector for shrew HTTP flood attacks. We consider the file popularity matrix as an $N$-dimensional stochastic process which is controlled by an underlying semi-Markov process. For an HsMM whose parameters are given, hidden state $s_{t}$ of the HsMM can be used to describe a representative spatial distribution of popularity of $N$ documents at $t^{\text {th }}$ time unit. Transitions of the hidden states (i.e., from $s_{t-1}$ to $s_{t}$ ) can be considered as the characteristics of access behaviors from one spatial distribution $\left(s_{t-1}\right)$ to another spatial distribution $\left(s_{t}\right)$. Duration $\left(\tau_{t}\right)$ of a hidden state $\left(s_{t}\right)$ can be considered as the time unit amount that the current spatial distribution $\left(s_{t}\right)$ will persists.

Considering the HsMM will be very complex when the observation is a multidimensional vector with dependent elements, we use the Principal component analysis [8] to reduce the dimensionality of file popularity matrix and apply the Independent component analysis [9] to obtain independent vector.

\subsection{PCA and ICA}

PCA is a well-established technique for dimensionality reduction and multivariate analysis. Examples of its many applications include data compression, image processing, visualization, exploratory data analysis, pattern recognition, time series prediction and distributed sensor network.

PCA aims to find a linear orthogonal transformation:

$$
\vec{y}_{t}=E \vec{a}_{t},
$$

where the rows of $E$ are the eigenvectors of the covariance matrix of the original $N$-dimensional data $\vec{x}$ (assumed to have 0 mean, condition that can always be easily achieved by subtracting the mean estimated over the training set). The $j^{\text {th }}$ principal component (PC, the $j^{\text {th }}$ element of $\vec{y}_{t}$ ) of a vector $\vec{a}_{t}$ corresponds to its projection along 
the direction of the $j^{\text {th }}$ eigenvector of the data covariance matrix, and the principal components are non-correlated. The eigenvectors are ordered so that, if $\sigma_{i}^{2}$ is the eigenvalue related to the $i^{\text {th }}$ eigenvector, then $\sigma_{1}^{2} \geq \sigma_{2}^{2} \geq \cdots \geq \sigma_{m}^{2}$. The eigenvalue accounts for the data variance along the direction of the corresponding eigenvector. The first eigenvector indicates the direction of highest variance in the data, the second one the direction orthogonal to the first one with the highest variance and so on. Often the last eigenvectors account for very small variance and the corresponding principal components can be eliminated. Thus, we choose the first $K$ eigenvectors having the largest eigenvalues. This implies that $K$ is the inherent dimensionality of the subspace governing the "signal" while the remaining $(N-K)$ dimensions generally contain noise. The dimensionality of the subspace $K$ can be determined by:

$$
\sum_{i=1}^{K} \lambda_{i} / \sum_{i=1}^{N} \lambda_{i} \geq \alpha
$$

where $\alpha$ is the ratio of variation in the subspace to the total variation in the original space. This allows a reduction of the dimensionality making the following anomaly detector based on HsMM faster and better trainable (the number of parameters is reduced as it depends on the size of the observation vector). A complete discussion of PCA can be found in [8].

ICA is a new statistical signal processing technique which is widely used in blind source separation (BSS). In contrast to the PCA which is sensitive to high-order relationships, the basic idea of ICA is to represent a set of random variables using basis function, where the components are statistically independent and non-gaussianity as possible. The main aim of ICA is to find the linear transformation:

$$
\vec{y}_{t}=W \vec{a}_{t},
$$

where $W$ is called unmixing matrix which satisfies $p\left(y_{i}, y_{j}\right)=p\left(y_{i}\right) p\left(y_{j}\right)$ for $i \neq j$, where $y_{i}$ is the $i^{\text {th }}$ element of $\vec{y}_{t}$. Many algorithms can be used to solve this non-Gaussian problem of ICA. This paper uses the FastICA algorithm [9] which has good performance in simulations and fast convergence during the parameter reestimate.

\subsection{Detection Architecture}

The overall procedure of our detection architecture is illustrated in Fig. 1. The scheme includes training phase and detection phase. The training phase includes the following five steps: (i) compute the file popularities of each document; (ii) input the file popularity matrix into PCA module and compute the orthogonal transformation $E$ and PCs; (iii) remain the first $K$ PCs by (5), $\alpha=80 \%$ in this paper; (iv) input the first $K$ PCs into the ICA module and compute the unmixing matrix $W$ and ICs; and (v) use the ICs to estimate the parameters of HsMM.

The detection is implemented as the follows: (i) compute the file popularities of each document; (ii) compute the first $K$ PCs of the observed data based on $E$; (iii) compute the ICs of the first $K$ PCs based on $W$; and (iv) ompute the entropy of observed data fitting to the HsMM by (2) and (3); 


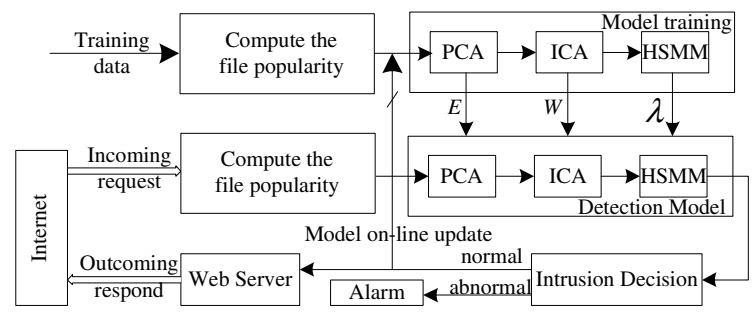

Fig. 1. Anomaly detector

In the practical implementation, the model is first trained by the stable and low volume Web workload whose normality can be ensured by most existing anomaly detection systems, and then it is used to monitor the following Web workload for a period of $T$ minutes. When the period is past, model will be updated by the new collected Web workload whose normality is ensured by its entropy fitting to the model. After the update, the model enters the next cycle for App-DDoS attacks detection. Web traffic is reported as anomalous if the difference between its entropy and normal level is larger than the predefined threshold

\section{Experiments}

We use the trace of FIFA 1998 WorldCup (http://ita.ee.lbl.gov/html/contrib /WorldCup.html) and emulate shrew HTTP flood attacks to validate the model.

In contrast to the traditional shrew attacks with constant attack parameters, we emulate a more stealthy type of shrew attacks of HTTP flood whose attack parameters are all chosen randomly by attack nodes. As shown in Fig. 2, a single source stochastic pulsing attack is modeled as a square waveform HTTP request stream with an attack period of $T$, length of the burst $l$, and the burst rate $H$. Among the variables, $T, l, H$ and $\Delta t$ are stochastic variables reset by each attack node randomly before it is going to fire the next pulsing attack, which makes the different attack nodes have different attack parameters and produces different attack parameters at different attack period. Thus, the attacks exhibit a fluctuating rate oscillating between different $H$ and zero and dynamics of a pulsing attack appear as a stochastic ON/OFF pattern.

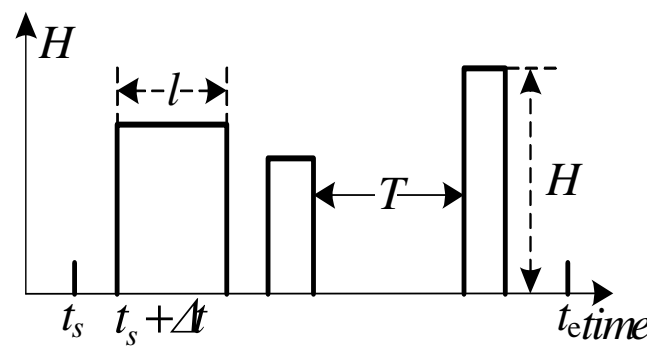

Fig. 2. Shrew HTTP flood attack

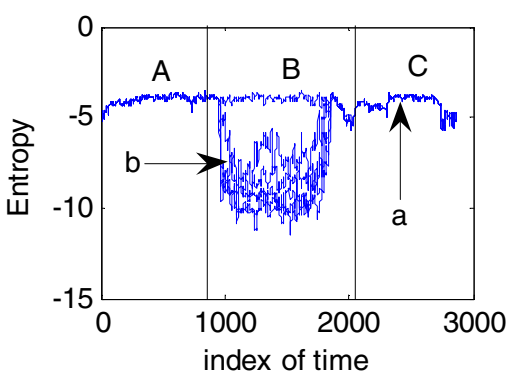

Fig. 3. Pulsing rate attack 


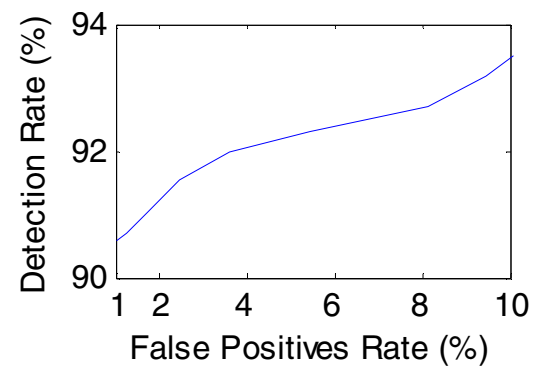

Fig. 4. ROC curve



Fig. 5. Entropy, DR and FPR

Fig. 3 shows the entropy varies with the time, where curve $a$ represents the entropy of normal flash crowd and curve cluster $b$ represents the entropy of normal flash crowd embedded with shrew HTTP flood attacks in zone $C$. As shown in the figure, although the flash crowd is burst and high volume, the document popularity is stable because of the steady access behavior of individual Web surfer, thus, the entropy series is stable. But, when the vicious requests are added into the flash crowd, the original popularity distribution of documents is destroyed, which cause the entropy series deviates from the normal level. Thus, the potential shrew HTTP flood attacks can be detected by the entropy of document popularity fitting to the model.

Fig. 4 is the receiver operating characteristics (ROC) curve showing the performance of our detection model. Fig. 5 shows, if we take -5.3 for threshold value of normal Web traffic's average entropy, the False Negative Ratio (FNR) is about 1\%, and the Detection rate is about $90 \%$.

\section{Conclusions and Future Work}

Based on PCA, ICA and HsMM, this paper proposes a novel proposal to detect the shrew HTTP flood attacks occurring during the flash crowd by monitoring the file popularity of Web server. Experiment results based on a real traffic and emulated attacks show the model could capture shift of Web traffic caused by the attacks occurring during flash crowd and might be both practical and helpful for triggering more focused detection and filtering in victim network.

One disadvantage of our scheme is the technique cannot readily distinguish and filter the malicious sources from the normal ones, which make it merely serve as an alert function to trigger more detailed monitoring mechanisms. We will improve it in our further work.

\section{Acknowledgments}

This work was supported by National Natural Science Foundation of China under grant no. 90304011, Guangdong Natural Science Foundation under grant no. 04009747 and Higher Education Foundation for Ph.D Program under grant no. 20040558043. 


\section{References}

1. Common Vulnerabilities and Exposures. http://www.cve.mitre.org/, 2005

2. S. Ranjan, R. Swaminathan, M. Uysal, and E. Knightly. "DDoS-Resilient Scheduling to Counter Application Layer Attacks under Imperfect Detection" in Proceedings of IEEE INFOCOM. April 2006. online: http://www-ece.rice.edu/ networks/papers/dos- sched.pdf

3. M. Almgren, U. Lindqvist, "Application-integrated data collection for security monitoring" in Proceedings of Recent Advances in Intrusion Detection, Davis, CA, October 2001, LNCS, Springer, 2001, pp.22-36.

4. G. Vigna, W. Robertson, V. Kher, R.A. Kemmerer, "A stateful intrusion detection system for world-wide Web servers" in the Proceedings of the Annual Computer Security Applications Conference, Las Vegas, NV, December 2003, pp. 34-43.

5. Juan M. Estévez-Tapiador, Pedro García-Teodoro, Jesús E. Díaz-Verdejo. "Detection of Web-based Attacks through Markovian Protocol Parsing" in the Proceedings of the 10th IEEE Symposium on Computers and Communications.

6. E. Tombini, H. Debar, L. Me, and M. Ducasse. "A Serial Combination of Anomaly and Misuse IDSes Applied to HTTP Traffic" In Proceedings of the Twentieth Annual Computer Security Applications Conference, Tucson, Arizona, December 2004.

7. Venkata N. Padmanabhan and Lili Qiu. "The content and access dynamics of a busy Web site: Findings and implications" In the proceeding of ACM SIGCOMM 2000, Stockholm, Sweden, Aug. 2000.

8. Lindsay I Smith. "A tutorial on Principal Components Analysis" [EB/OL]. http://www.snl.salk.edu/ shlens/pub/ notes/pca.pdf, 2003-03.

9. A. Hyvärinen. "Survey on Independent Component Analysis" Neural Computing Surveys, 1999,2. pp.94-128.

10. S.-Z. Yu, and H. Kobayashi, "An Efficient Forward-Backward Algorithm for an Explicit Duration Hidden Markov Model” IEEE Signal Processing Letters, Vol. 10, No. 1, January 2003, pp. 11-14.

11. S. Kandula, D. Katabi, M. Jacob, and A. W. Berger. "Botz-4-sale: Surviving organized DDoS attacks that mimic flash crowds" Technical Report TR-969, MIT., October 2004. Online: http://www.usenix.org/events/nsdi05/tech /kandula/kandula.pdf

12. G.Mori and J.Malik. "Recognizing objects in adversarial clutter: Breaking a visual captcha" IEEE Computer Vision and Pattern Recognition, 2003.

13. X. Luo, and R. K. C. Chang, "On a New Class of Pulsing Denial-of-Service Attacks and the defense," Network and Distributed System Security Symposium (NDSS'05), San Diego, CA., Feb. 2-5, 2005. 